\title{
SEARCH FOR PULSED TeV GAMMA-RAY EMISSION FROM THE CRAB PULSAR
}

\author{
R. W. Lessard, ${ }^{1}$ I. H. Bond,${ }^{2}$ S. M. Bradbury, ${ }^{2}$ J. H. Buckley, ${ }^{3}$ A. M. Burdett, ${ }^{2,4}$ D. A. Carter-Lewis, ${ }^{5}$ \\ M. Catanese, ${ }^{5}$ M. F. Cawley, ${ }^{6}$ M. D'Vali, ${ }^{2}$ D. J. Fegan, ${ }^{7}$ J. P. Finley, ${ }^{1}$ J. A. Gaidos, ${ }^{1}$ G. H. Gillanders, ${ }^{8}$ \\ T. Hall ${ }^{1}$ A. M. Hillas, ${ }^{2}$ F. Krennrich, ${ }^{5}$ M. J. Lang, ${ }^{8}$ C. Masterson, ${ }^{7}$ P. Moriarty, ${ }^{9}$ J. Quinn, ${ }^{7}$ H. J. Rose, ${ }^{2}$ \\ F. W. Samuelson, ${ }^{5}$ G. H. Sembroski, ${ }^{1}$ R. Srinivasan, ${ }^{1}$ V. V. Vassiliev, ${ }^{4}$ \\ AND T. C. WEEKES ${ }^{4}$ \\ Received 1999 June 8; accepted 1999 October 18
}

\begin{abstract}
We present the results of a search for pulsed TeV emission from the Crab pulsar using the Whipple Observatory's $10-\mathrm{m}$ gamma-ray telescope. The direction of the Crab pulsar was observed for a total of $73.4 \mathrm{hr}$ between 1994 November and 1997 March. During this period the Whipple $10 \mathrm{~m}$ telescope was operated at its lowest energy threshold to date. Spectral analysis techniques were applied to search for the presence of a gamma-ray signal from the Crab pulsar over the energy band $250 \mathrm{GeV}$ to $4 \mathrm{TeV}$. We do not see any evidence of the $33 \mathrm{~ms}$ pulsations present in other energy bands from the Crab pulsar. The 99.9\% confidence level upper limit for pulsed emission above $250 \mathrm{GeV}$ is derived to be $4.8 \times 10^{-12}$ $\mathrm{cm}^{-2} \mathrm{~s}^{-1}$ or less than $3 \%$ of the steady flux from the Crab Nebula. These results imply a sharp cutoff of the power-law spectrum seen by the EGRET instrument on the Compton Gamma-Ray Observatory. If the cutoff is exponential, it must begin at $60 \mathrm{GeV}$ or lower to accommodate these upper limits.
\end{abstract}

Subject headings: gamma rays: observations - pulsars: individual (Crab Pulsar)

\section{INTRODUCTION}

The Crab pulsar/Nebula system is one of the most intensely studied astrophysical sources with measurements throughout the electromagnetic spectrum from the radio to the $\mathrm{TeV}$ energy band. In most regions of the spectrum, the characteristic $33 \mathrm{~ms}$ pulsations of the pulsar are clearly visible. The pulse profile is unique among known pulsars in that it is aligned from radio to gamma-ray energies. The study of the pulsed emission in different energy ranges is of considerable importance to understanding the underlying emission mechanisms (e.g., Eikenberry \& Fazio 1997). The EGRET instrument on the Compton Gamma-Ray Observatory $(C G R O)$ has shown that there is pulsed gamma-ray emission from the pulsar up to at least $10 \mathrm{GeV}$ (Ramanamurthy et al. 1995). Current imaging atmospheric Cerenkov telescopes have firmly established the Crab Nebula as a steady source of gamma rays from $300 \mathrm{GeV}$ to $50 \mathrm{TeV}$ (Hillas et al. 1998; Tanimori et al. 1998). However, these observations have not detected any significant modulation of this TeV signal at the period of the pulsar. In contrast to these reports, other groups have reported $\mathrm{TeV}$ emission modulated at the $33 \mathrm{~ms}$ period of the Crab pulsar. Some of these reports have been associated with episodic

\footnotetext{
${ }^{1}$ Department of Physics, Purdue University, West Lafayette, IN 47907; lessard@physics.purdue.edu.

${ }^{2}$ Department of Physics, University of Leeds, Leeds, LS2 9JT, U.K.

${ }^{3}$ Department of Physics, Washington University, St. Louis, MO 63130.

${ }^{4}$ Whipple Observatory, Harvard-Smithsonian CfA, P.O. Box 97, Amado, AZ 85645-0097.

${ }^{5}$ Department of Physics and Astronomy, Iowa State University, Ames, IA 50011.

${ }^{6}$ Department of Physics, National University of Ireland, Maynooth, Co. Kildare, Ireland.

${ }^{7}$ Department of Experimental Physics, University College Dublin, Belfield, Dublin 4, Ireland.

${ }^{8}$ Department of Physics, National University of Ireland, Galway, Ireland.

${ }^{9}$ School of Science, Galway-Mayo Institute of Technology, Galway, Ireland.
}

activity (Gibson et al. 1982; Bhat et al. 1986; Acharya et al. 1992). A persistent pulsed signal from the Crab pulsar was reported by the Durham group (Dowthwaite et al. 1984). However, this has not been confirmed by more sensitive observations that show that less than $5 \%$ of the total very high energy (VHE) flux is pulsed (Weekes et al. 1989; Reynolds et al. 1993; Goret et al. 1993). At ultrahigh energies, the CASA-MIA experiment does not find any statistically significant evidence for pulsed gamma-ray emission at the Crab pulsar period, on an interval of 1 day or longer, based on the analysis of data recorded during the interval 1990 March to 1995 October (Borione et al. 1997).

Pulsed emission from the Crab pulsar at IR energies and above is generally believed to originate in the magnetosphere of the system far from the stellar surface. In each of the two models that address the pulsed gamma-ray emission in detail, the outer gap model (Cheng, Ho, \& Ruderman 1986; Romani 1996) and the polar cap model (Daugherty \& Harding 1982), the high-energy flux arises from curvature radiation of pairs as they propagate along the open field lines of the magnetosphere. The specific details of the pulse shapes in different pulsars are explained by the line-of-sight geometry of the observer relative to the spin and magnetic axes of the rotating neutron star in these models. The energy at which the pulsed flux begins to cut off and the detailed spectral shape of the cutoff can help to distinguish between the two models. Given the detection of pulsations out to $10 \mathrm{GeV}$ by EGRET (Ramanamurthy et al. 1995) and the restrictive upper limits above $300 \mathrm{GeV}$ (Weekes et al. 1989; Reynolds et al. 1993; Goret et al. 1993), the cutoff necessarily resides in the $\sim 100 \mathrm{GeV}$ energy range. This is our primary motivation for this deep search for pulsations from the Crab in the $100 \mathrm{GeV}$ range.

The outer gap model by Romani (1996) also includes TeV emission via the synchrotron-self-Compton mechanism that produces a peak spectral energy density above $1 \mathrm{TeV}$. Such a mechanism could in principle explain the detection of pulsed emission by the Durham group, which operates at an energy threshold of $1 \mathrm{TeV}$, and still be consistent with 
TABLE 1

Definition of the Image Parameters, Which Are Used to Characterize the Image Shape and Orientation (See Fig. 1)

\begin{tabular}{|c|c|}
\hline Parameter & Definition \\
\hline $\max 1$ & Largest signal recorded by the PMTs \\
\hline $\max 2 \ldots \ldots \ldots$ & Second largest signal recorded by the PMTs \\
\hline Size ........... & Sum of all signals recorded \\
\hline Width ........ & The root mean square (rms) spread of light along the minor axis of the image; a measure of the lateral development of the shower \\
\hline Length ....... & The rms spread of light along the major axis of the image; a measure of the vertical development of the shower. \\
\hline Distance...... & The distance from the centroid of the image to the center of the field of view \\
\hline$\alpha \ldots \ldots \ldots \ldots$ & The angle between the major axis of the image and a line joining the centroid of the image to the center of the field of view \\
\hline
\end{tabular}

the upper limits reported at lower energies. For this reason we have applied spectral analysis techniques to search for a gamma-ray Crab pulsar signal over the energy band 250 $\mathrm{GeV}$ to $4 \mathrm{TeV}$.

\section{OBSERVATION AND ANALYSIS TECHNIQUES}

The VHE observations reported in this paper utilize the atmospheric Cerenkov technique (Cawley \& Weekes 1995) and the 10-m optical reflector located at the Whipple Observatory on Mount Hopkins in southern Arizona (elevation $2.3 \mathrm{~km}$ ) (Cawley et al. 1990). A camera, consisting of photomultiplier tubes (PMTs) mounted in the focal plane of the reflector, detects the Cerenkov radiation produced by gamma-ray and cosmic-ray air showers from which an image of the Cerenkov light can be reconstructed. For most of the observations reported here, the camera consisted of 109 PMTs (each viewing a circular field of 0.259 radius) with a total field of view of $3^{\circ}$ in diameter. In December 1996, 42 additional PMTs were added to the camera, increasing the field of view to 3.3 .

We characterize each Cerenkov image using a moment analysis (Reynolds et al. 1993). The roughly elliptical shape of the image is described by the length and width parameters, and its location and orientation within the field of view are given by the distance and $\alpha$ parameters, respectively. We also determine the two highest signals recorded by the PMTs (max1, max2) and the amount of light in the image (size). These parameters are defined in Table 1 and are depicted in Figure 1. Gamma-ray events give rise to more compact shower images than background hadronic showers and are preferentially oriented toward the putative source position in the image plane. By making use of these differences, a gamma-ray signal can be extracted from the large background of hadronic showers.

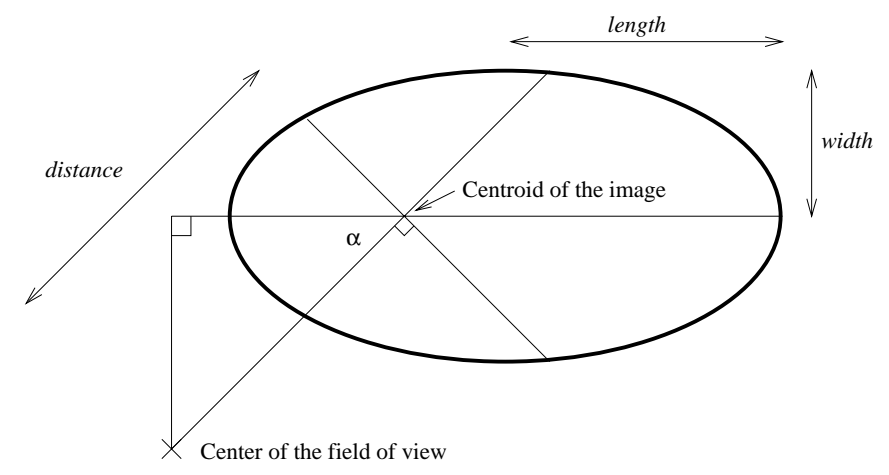

FIG. 1.- Images of Cerenkov light produced by gamma-ray and cosmic-ray-induced air showers are parameterized using a moment analysis to describe the image shape and orientation in the image plane.

\subsection{Selection Methods}

The standard gamma-ray selection method utilized by the Whipple Collaboration is the Supercuts criteria (see Table 2; cf. Reynolds et al. 1993; Catanese et al. 1996). These criteria were optimized on contemporaneous Crab Nebula data to give the best sensitivity to point sources. In an effort to remove the background of events triggered by single muons and night-sky fluctuations, Supercuts incorporates preselection cuts on the size and on $\max 1$ and $\max 2$. While the introduction of a preselection is desirable from the point of view of optimizing overall sensitivity, it automatically rejects many showers below $\sim 400 \mathrm{GeV}$. In the context of a search for pulsed emission from the Crab pulsar, which must have a low-energy cutoff to accommodate existing upper limits, this is clearly undesirable. Accordingly, a modified set of cuts (Table 3; cf. Moriarty et al. 1997), developed to provide optimal sensitivity in the $\sim 200 \mathrm{GeV}$ to $\sim 400 \mathrm{GeV}$ region and referred to hereafter as Smallcuts, was used for the events that failed the Super-

TABLE 2

SuPERCUts Gamma-Ray Selection Criteria

\begin{tabular}{|c|c|}
\hline Supercuts $(1995 / 1996)$ & Supercuts (1997) \\
\hline \multicolumn{2}{|c|}{ Preselection Criteria } \\
\hline $\begin{aligned} \max 1 & >100 \text { d.c. } \\
\max 2 & >80 \text { d.c. } \\
\text { size } & >400 \text { d.c. }\end{aligned}$ & $\begin{array}{c}\max 1>95 \text { d.c. } \\
\max 2>45 \text { d.c. } \\
\text { size }>0 \text { d.c. }\end{array}$ \\
\hline \multicolumn{2}{|c|}{ Gamma-Ray Selection } \\
\hline $\begin{array}{c}0.073<\text { width }<0.15 \\
0.16<\text { length }<0.30 \\
0.51<\text { distance }<1.10 \\
\alpha<15^{\circ}\end{array}$ & $\begin{array}{c}0.073<\text { width }<0.16 \\
0.16<\text { length }<0.33 \\
0.51<\text { distance }<1.17 \\
\alpha<15^{\circ}\end{array}$ \\
\hline
\end{tabular}

${ }^{a}$ d.c. $=$ digital counts $(1.0$ d.c. $\approx 1.0$ photoelectron $)$.

TABLE 3

Smallcuts Gamma-Ray Selection Criteria Applied to Events Which Failed the Supercuts Preselection Criteria

\begin{tabular}{c|c}
\hline \hline Smallcuts $(1995 / 1996)$ & Smallcuts $(1997)$ \\
\hline $\max 1>40$ d.c. & $\max 1>40$ d.c. \\
$\max 2>40$ d.c. & $\max 2>40$ d.c. \\
size $>0$ d.c. & size $>0$ d.c. \\
length/size $<8.3 \times 10^{-4}$ d.c. $^{-1}$ & length $/$ size $<8.3 \times 10^{-4}$ d.c. $^{-1}$ \\
$0.073<$ width $<0.13$ & $0.073<$ width $<0.13$ \\
$0.16<$ length $<0.30$ & $0.16<$ length $<0.33$ \\
$0.51<$ distance $<1.10$ & $0.51<$ distance $<1.17$ \\
$\alpha<15^{\circ}$ & $\alpha<15^{\circ}$ \\
\hline
\end{tabular}




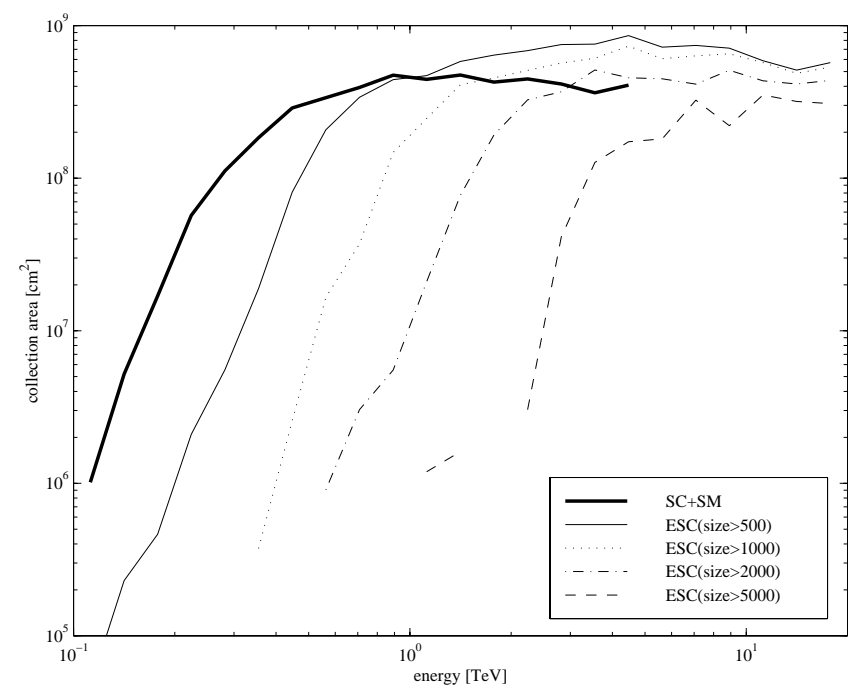

FIG. 2.-Collection areas for Supercuts combined with Smallcuts $(\mathrm{SC}+\mathrm{SM})$ and Extended Supercuts (ESC) combined with a lower bound on image size, which selects only the higher energy events.

cuts preselection criteria. The most notable difference between Smallcuts and Supercuts is the introduction of a cut on the length/size of an image. Such a cut is effective at discriminating partial arcs of Cerenkov light rings arising from single muons, which become the predominant background at lower energies. These images tend to be long
TABLE 4

EXTENDED SUPERCUTS

\begin{tabular}{c}
\hline \hline Extended Supercuts $(1995 / 1997)$ \\
\hline $\max 1>70$ d.c. \\
$\max 2>70$ d.c. \\
size $>500,1000,2000,5000$ d.c. \\
$\mid$ width $+0.022-0.023 \ln ($ size $) \mid<0.048$ \\
$\mid$ length $-0.114-0.020 \ln ($ size $) \mid<0.068$ \\
$0.6<$ distance $<1.0$ \\
$\alpha-9.16+0.558 \ln ($ size $)<13.5$ \\
\hline
\end{tabular}

compared to their intensity and so may be rejected on the basis of the length/size ratio. When a combination of Supercuts and Smallcuts is used, Monte Carlo simulations indicate that this analysis results in an energy threshold of $\sim 250 \mathrm{GeV}$. This threshold is the energy at which the differential rate from a source with a spectral index equal to that of the steady Crab Nebula reaches its peak. The collection area as a function of gamma-ray energy is depicted in Figure 2 and results in an effective collection area of $2.7 \times 10^{8} \mathrm{~cm}^{2}$. Details of the methods used to estimate the energy threshold and effective area are given elsewhere (Mohanty et al. 1998).

The data from 1997 were analyzed with slightly modified cuts (see Tables 2 and 3), which were reoptimized after an upgrade to the Whipple camera, which increased the field of view. The greatest effect of the larger field of view was that
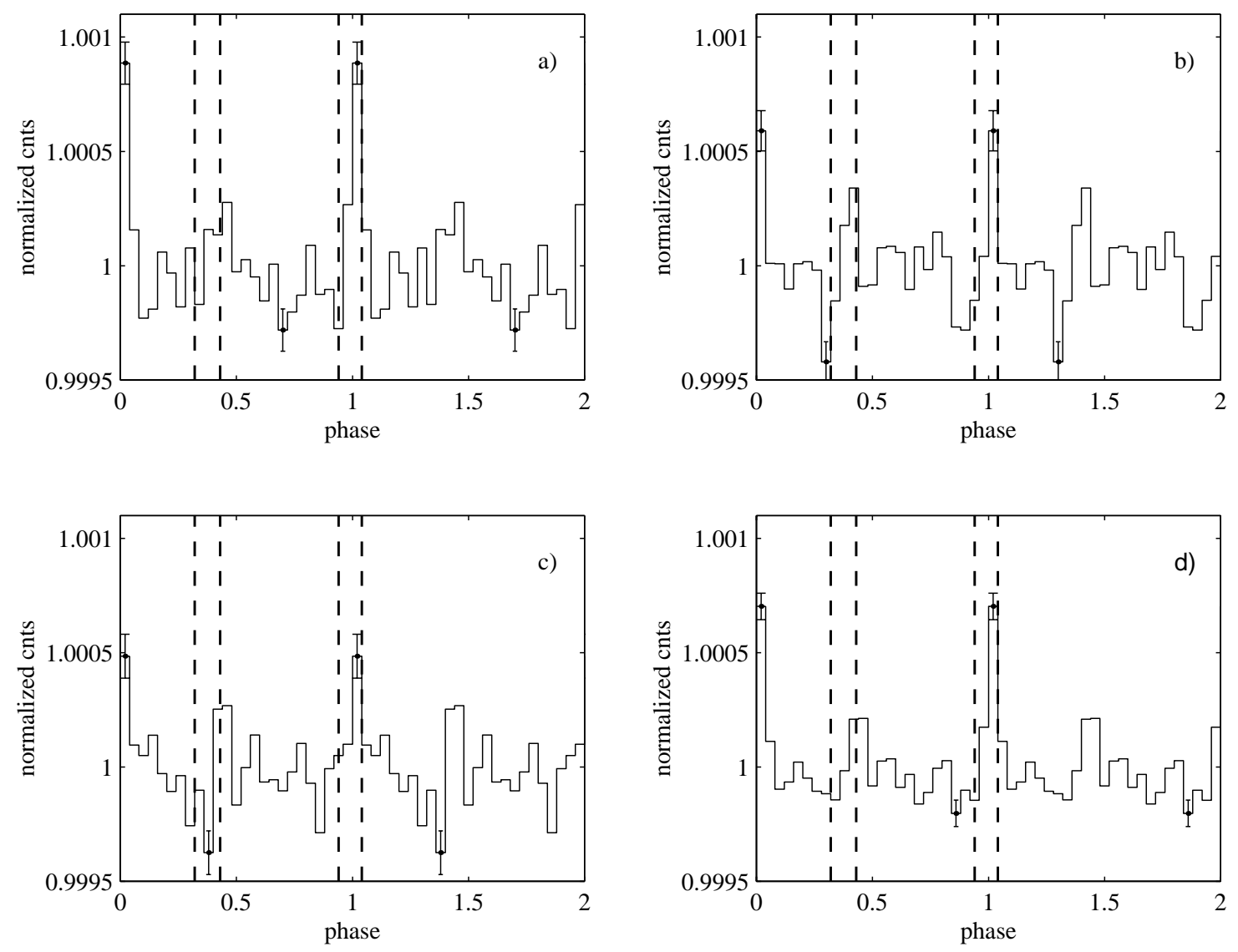

FIG. 3.-Optical observations of the Crab pulsar with the Whipple 10-m telescope. The data sets show a clear detection of the Crab optical pulsations. Phase 0.0 (in each case) corresponds to the extrapolated arrival of the radio peak closest to the epoch of observations derived from the Jodrell Bank timing solution. The counts in each case were normalized to the average of the respective observation. (a) Data set taken on 1996 December 2 (UT); (b) data set taken on 1996 December 18 (UT); (c) data set taken on 1997 March 11 (UT); (d) addition of the above data sets (in phase). The dashed lines depicts the EGRET main and intrapulse phase ranges. 
TABLE 5

SElected Events for Periodic ANAlysis

\begin{tabular}{lrrr}
\hline \hline Selection & $N_{\text {on }}$ & $N_{\text {off }}$ & Significance $(\sigma)$ \\
\hline Supercuts + Smallcuts $\ldots \ldots \ldots \ldots \ldots \ldots$ & 6696 & 6636 & 0.65 \\
Extended Supercuts $($ size $>500) \ldots \ldots \ldots$ & 4709 & 4748 & -0.50 \\
Extended Supercuts $(\operatorname{size}>1000) \ldots \ldots$ & 1738 & 1762 & -0.51 \\
Extended Supercuts $($ size $>2000) \ldots \ldots$ & 602 & 649 & -1.67 \\
Extended Supercuts $($ size $>5000) \ldots \ldots$ & 125 & 150 & -1.88 \\
\hline
\end{tabular}

NotE. $-N_{\text {on }}$ are the number of events with phases within the EGRET pulse profile, and $N_{\text {off }}$ are the background estimated from events falling outside the EGRET pulse profile. images appeared longer and at a greater distance from the center of the field of view owing to less image truncation than caused by the smaller camera.

Supercuts was optimized to give the best point-source sensitivity, but in doing so it rejects many of the larger gamma-ray events. Another selection process, known as Extended Supercuts (Table 4; cf. Mohanty et al. 1998), was utilized to facilitate a search for pulsed emission over the energy band $250 \mathrm{GeV}$ to $4 \mathrm{TeV}$. This method is quite similar to Supercuts but scales the various cuts with the shower size and retains approximately $95 \%$ of gamma-ray events compared to approximately $50 \%$ of gamma-ray events passed
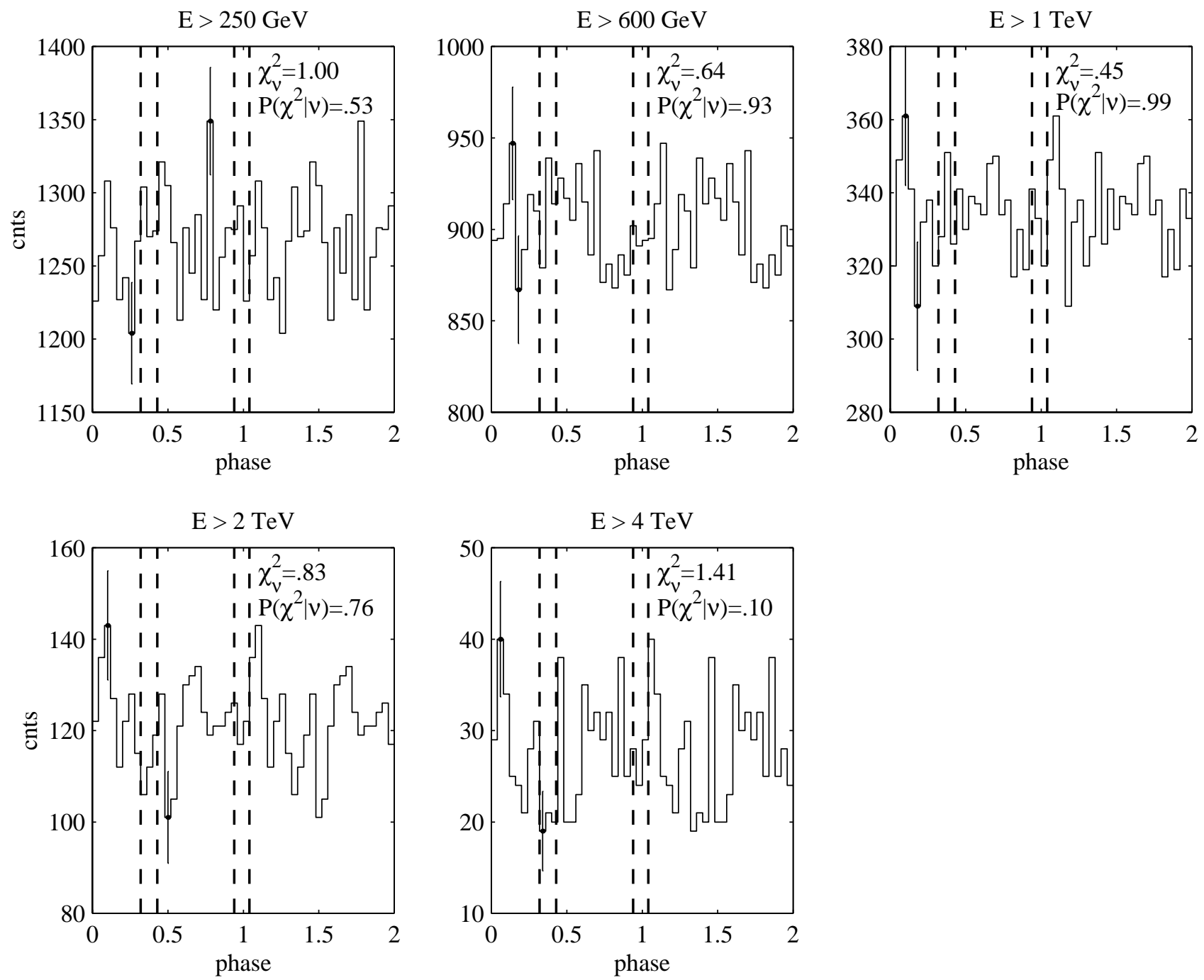

FIG. 4. - Search for TeV gamma rays from the Crab pulsar. The dashed lines depict the EGRET main pulse and intrapulse phase ranges. Error bars have been included on bins with the maximum and minimum number of counts. The $\chi^{2}$ probability that each distribution is consistent with its mean is given in each panel. We find no evidence of pulsed emission at the radio period.

TABLE 6

INTEGRAL FLUX UPPER LIMITS

\begin{tabular}{ccc}
\hline \hline Selection Method & Periodic Emission $\left(\mathrm{cm}^{-2} \mathrm{~s}^{-1}\right) \times 10^{-13}$ & Threshold $(\mathrm{TeV})$ \\
\hline Supercuts + Smallcuts $\ldots \ldots \ldots \ldots \ldots \ldots$ & $<48.2$ & $\geq 0.25$ \\
Extended Supercuts $($ size $>500) \ldots \ldots$. & $<16.7$ & $\geq 0.6$ \\
Extended Supercuts $($ size $>1000) \ldots \ldots$ & $<12.0$ & $\geq 1.0$ \\
Extended Supercuts $($ size $>2000) \ldots \ldots$ & $<5.9$ & $\geq 2.0$ \\
Extended Supercuts $($ size $>5000) \ldots \ldots$ & $<4.6$ & $\geq 4.0$ \\
\hline
\end{tabular}




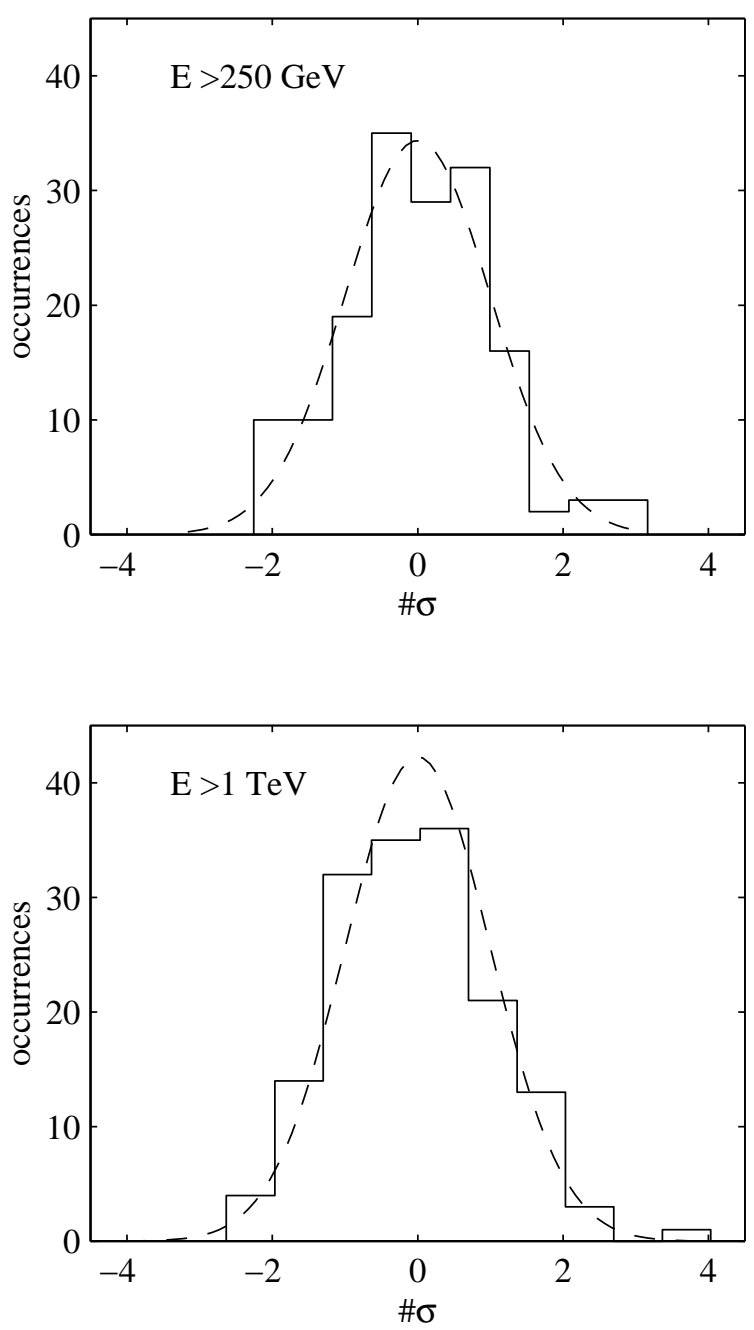

FIG. 5.-Left-hand panels: Statistical significance of excess events for each observation for energy thresholds of $250 \mathrm{GeV}$ and $1 \mathrm{TeV}$. Right-hand panels: Corresponding distributions of significances as solid lines. The dashed curves shows the statistical expectation for zero excess.

by the Supercuts criteria. By applying a lower bound on the size of an image, the energy threshold of the analysis increases. Figure 2 depicts the collection area as a function of gamma-ray energy as derived by Monte Carlo simulations for a lower bound on the size of an image of 500,1000, 2000, and 5000 digital counts. These cuts impose energy thresholds of $0.6,1.0,2.0$, and $4.0 \mathrm{TeV}$, respectively.

\subsection{Periodic Analysis}

The arrival times of the Cerenkov events were registered by a Global Positioning System (GPS) clock with an absolute resolution of $250 \mu \mathrm{s}$. An oscillator, calibrated by GPS second marks (relative resolution of $100 \mathrm{~ns}$ ), was used to interpolate to a resolution of $0.1 \mu \mathrm{s}$. After an oscillator calibration was applied, all arrival times were transformed to the solar system barycenter by utilizing the JPL DE200 ephemeris as described by Standish (1982). As the acceleration of the pulsar relative to the solar system barycenter is negligible, the only additional correction factor is due to the gravitational redshift. The conversion of the coordinated universal time (UTC) as measured at the telescope, to the solar system barycenter arrival time (TDB), is given by

$$
t_{\mathrm{TDB}}=t_{\mathrm{UTC}}+\Delta_{\mathrm{TAI}-\mathrm{UTC}}+\Delta_{\mathrm{TDT}-\mathrm{TAI}}+\Delta_{\mathrm{TDB}-\mathrm{TDT}}+\Delta_{\mathrm{REL}} \text {. }
$$

The international atomic time (TAI) differs from UTC time by an integral number of leap seconds. The terrestrial dynamical time (TDT) is used as a timescale of ephemerides for observations from the Earth's surface and differs from TAI by $32.184 \mathrm{~s}$. The correction to the Earth's surface requires the telescope's geocentric coordinates and a model of the Earth's motion. The final correction applied, $\Delta_{\text {REL }}$, accounts for the variation of the gravitational potential around the Earth's orbit.

The corrected times were folded to produce the phases, $\phi_{j}$, of the events modulo the pulse period according to

$$
\phi_{j}=\phi_{0}+v\left(t_{j}-t_{0}\right)+\frac{1}{2} \dot{v}\left(t_{j}-t_{0}\right)^{2},
$$

where $v, \dot{v}$ are the frequency and first frequency derivative at the epoch of observation $t_{0}$. For each source run the valid frequency parameters were derived from the J2000 ephemeris obtained from Jodrell Bank, where the Crab pulsar is monitored on a monthly basis.

To check the Whipple Observatory timing systems an optical observation of the Crab pulsar was undertaken on the nights of 1996 December 2 (UT), 1996 December 18 (UT), and 1997 March 11 (UT), using the 10-m reflector with a photometer at its focus (Srinivasan et al. 1997). The signal from the photometer was recorded by the data acquisition electronics and timing system of the telescope, 


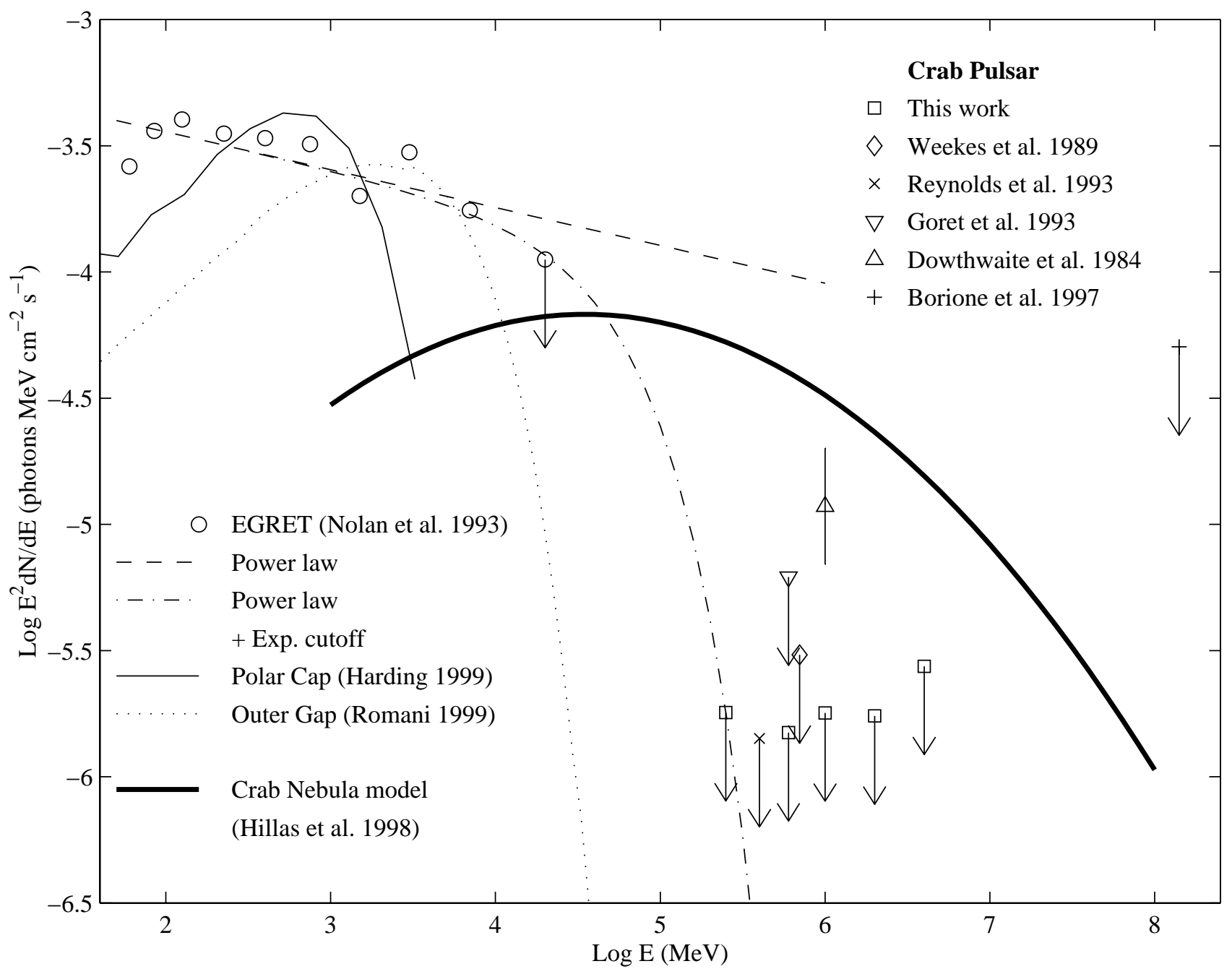

FIG. 6. - Pulsed photon spectrum of the Crab pulsar. The EGRET data points are from Nolan et al. (1993). The thin solid line is the polar cap model fit to the EGRET data (A. K. Harding 1999, private communication). The dotted line is the outer gap model for the Vela pulsar (scaled to match the EGRET Crab pulsar flux at peak intensity) and is included to indicate the shape of the cutoff this model predicts (R. W. Romani 1999, private communication). The dashed line represents the power-law fit to the EGRET data (Nolan et al. 1993). The dot-dashed line represents eq. (3) with a cutoff energy $E_{0}=60 \mathrm{GeV}$. The upper limits for pulsed emission presented in this paper are represented by the open squares. The thick solid curve depicts the model of unpulsed GeV-TeV emission from the Crab Nebula (Hillas et al. 1998).

thereby providing a direct test of the instrument's timing characteristics. The phase analysis of the event arrival times, depicted in Figure 3, yielded a clear detection of the optical signal from the Crab pulsar in phase with the radio pulse. This demonstrates the validity of the timing, data acquisition, and barycentering software in the presence of a pulsed signal.

\section{OBSERVATIONS AND RESULTS}

The position of the Crab pulsar was observed between 1995 January and 1997 March. The traditional mode of observing potential periodic sources with the Whipple Observatory gamma-ray telescope is to track the putative source location continuously for runs of 28 minute duration. After filtering runs for bad weather and instrumental problems, the data set consists of 159 runs for a total source observing time of $73.4 \mathrm{hr}$. The radio position (J2000) of the Crab pulsar $\left(\alpha=05^{\mathrm{h}} 34^{\mathrm{m}} 31^{\mathrm{s}} .949, \quad \delta=22^{\circ} 00^{\prime} 52^{\prime \prime} .057\right)$ was assumed for the subsequent timing analysis.

The numbers of events passing the selection criteria described above are given in Table 5. The phases of these events, shown in Figure 4, are used for periodic analysis. We find no evidence of pulsed emission at the radio period. To calculate upper limits for pulsed emission we have used the pulse profile seen at lower energies by EGRET. That is, we assume emission occurs within the phase ranges of both the main pulse, phase 0.94-0.04, and the intrapulse, phase 0.320.43 (Fierro et al. 1998). The number of events with phases within these intervals constitutes the number of candidate pulsed events, $N_{\text {on }}$. $N_{\text {off }}$, an estimate of the numbers of background events, is obtained by multiplying the number of events with phases outside these pulse intervals by the ratio of ranges spanned by the pulse and nonpulse regions. The results are given in Table 5. The statistical significance of the excess is calculated using the maximum likelihood method of $\mathrm{Li} \& \mathrm{Ma}$ (1983). The $99.9 \%$ confidence level upper limits calculated using the method of Helene (1983) are given in Table 6.

Several reports of pulsed emission from the Crab pulsar at very high energies claim to have seen evidence of episodic emission on time scales of several minutes. For this reason we have performed a run-by-run search for periodic emis- 
sion from the Crab pulsar based on the above pulse profile. The statistical significance of excess events for each observation and the corresponding distribution of significance for the lowest and middle energy ranges are given in Figure 5. In each energy band the distribution of significance is consist with the statistical expectation for zero excess.

\section{DISCUSSION}

Data taken with the Whipple Observatory's $10 \mathrm{~m}$ gamma-ray telescope have been used to search for pulsations from the Crab pulsar above $250 \mathrm{GeV}$. We find no evidence of pulsed emission at the radio period, and upper limits on the integral flux have been given.

To model the pulsed gamma-ray spectrum, a function of the form

$$
d N / d E=K E^{-\gamma} e^{-E / E_{o}}
$$

was used, where $E$ is the photon energy, $\gamma$ is the photon spectral index, and $E_{o}$ is the cutoff energy. The source spectrum in the EGRET energy range is well fitted by a power law with a photon spectral index of $-2.15 \pm 0.04$ (Nolan et al. 1993). The pulsed upper limit above $250 \mathrm{GeV}$ reported here is $\sim 3$ orders of magnitude below the flux predicted by the EGRET power law. Equation 3 was used to extrapolate the EGRET spectrum to higher energies constrained by the $\mathrm{TeV}$ upper limit reported here and indicates a cutoff energy $E_{o} \leq 60 \mathrm{GeV}$ for pulsed emission (see Fig. 6).

As indicated in $\S 2.1$, the energy threshold of the technique is derived assuming a source with a spectral index equal to that of the steady Crab Nebula. With the above model, this assumption is invalid. If we assume a source spectrum as given by equation (3) and define energy threshold and effective collection area as stated in $\S 2.1$, we simultaneously solve for an energy threshold of $180 \mathrm{GeV}$ and energy cutoff of $60 \mathrm{GeV}$. The derived cutoff energy is the same as that obtained assuming a Crab Nebula spectrum and indicates the robustness of defining the energy threshold of the technique in this way.

The sharpness of the spectral cutoff of the emission models depicted in Figure 6 provides a good discriminant. The status of current observations and the derived cutoff given above indicates that the cutoff must lie in the 10-60 $\mathrm{GeV}$ range. However, the upper limits reported here are well above the flux predicted by the polar cap and outer gap models and offer no discrimination between them. In contrast, the outer gap model of Romani (1996) predicts TeV emission via the synchrotron-self-Compton mechanism. The flux produced via this mechanism is dependent on the density and spectrum of primary electrons and positrons in the gap, as well as the density of local soft photon fields. The predicted pulsed $\mathrm{TeV}$ flux for a young gamma-ray pulsar is somewhat less than $1 \%$ of the pulsed GeV flux. The results reported here derive an upper limit to this fraction of less than $0.07 \%$.

We acknowledge the technical assistance of K. Harris, T. Lappin, and E. Roache. We thank A. Lyne and R. Pritchard for providing the radio ephemeris of the Crab pulsar. This research is supported by grants from the US Department of Energy, NASA, the Irish National Research Support Fund Board and by PPARC in the United Kingdom.
Acharya, B. S., et al. 1992, A\&A, 258, 412

Bhat, P. N., et al. 1986, Nature, 319, 127

Borione, A., et al. 1997, ApJ, 481, 313

Catanese, M., et al. 1996, in Towards a Major Atmospheric Cherenkov Detector - IV (Padova, Italy), ed. M. Cresti (Piazzola sul Brenta, Italy: Papergraf), 335

Cawley, M. F., et al. 1990, Exp. Astron., 1, 173

Cawley, M. F., \& Weekes, T. C. 1995, Exp. Astron., 6, 7

Cheng, K. S., Ho, C., \& Ruderman, M. 1986, ApJ, 300, 500

Daugherty, J. K., \& Harding, A. K. 1982, ApJ, 252, 337

Dowthwaite, J. C., et al. 1984, ApJ, 286, L35

Eikenberry, S. S., \& Fazio, G. G. 1997, ApJ, 476, 281

Fierro, J. M., et al. 1998, ApJ, 494, 734

Gibson, A. I., et al. 1982, Nature, 296, 833

Goret, P., et al. 1993, A\&A, 270, 401

\section{REFERENCES}

Helene, O. 1983, Nucl. Instrum. Methods, 212, 319

Hillas, A. M., et al. 1998, ApJ, 503, 744

Li, T. P., \& Ma, Y. Q. 1983, ApJ, 272, 317

Mohanty, G., et al. 1998, Astropart. Phys., 9, 15

Moriarty, P., et al. 1997, Astropart. Phys., 7, 315

Nolan, P. L., et al. 1993, ApJ, 409, 697

Ramanamurthy, P. V., et al. 1995, ApJ, 450, 791

Reynolds, P. T., et al. 1993, ApJ, 404, 206

Romani, R. W. 1996, ApJ, 470, 469

Srinivasan, R., et al. 1997, in Towards a Major Atmospheric Cherenkov Detector-V (Kruger Park, South Africa), ed. O. C. de Jager (Potchefstroom, South Africa: Westprint), 51

Standish, E. M., Jr. 1982, A\&A, 114, 297

Tanimori, T., et al. 1998, ApJ, 492, L33

Weekes, T. C., et al. 1989, ApJ, 342, 379 